\title{
Efeito da Temperatura Ambiente e da Restrição Alimentar sobre o Desempenho e a Composição de Fibras Musculares Esqueléticas de Frangos de Corte ${ }^{1}$
}

\author{
José Roberto Sartori², Elisabeth Gonzales ${ }^{3}$, Vitalino Dal Pai ${ }^{4}$, Henrique Nunes de Oliveira ${ }^{5}$, \\ Marcos Macari ${ }^{6}$
}

\begin{abstract}
RESUMO - O objetivo desta pesquisa foi estudar os efeitos da temperatura ambiente e da restrição alimentar sobre o desempenho e a composição do músculo flexor longo do hálux de frangos de corte. Trezentos e vinte e quatro pintos machos da linhagem Ross, com cinco dias de idade, foram distribuídos em um delineamento em parcelas subdivididas, considerando os tratamentos principais no esquema fatorial $3 \times 3$ inteiramente casualizado (três programas de alimentação: ad libitum, restrição precoce - 8 a 14 dias e restrição tardia - 29 a 35 dias; três níveis de temperatura: termoneutra, calor e frio). Os tratamentos secundários foram as idades das aves (seis idades: 7, 14, 21, 28, 35 e 42 dias). Não houve interação entre programa de alimentação e temperatura ou entre programa de alimentação, temperatura e idade para as características de desempenho dos frangos. Independentemente do programa de alimentação, houve efeito de temperatura para peso, ganho de peso, consumo de ração e conversão alimentar. O programa de alimentação afetou o peso e o ganho de peso das aves. Houve interação entre programa de alimentação e idade e entre temperatura e idade para peso vivo ao abate e peso do músculo da perna direita. Estas interações não foram significativas para peso do músculo da perna esquerda, área da secção transversal do músculo e relação peso do músculo/peso vivo ao abate. Conclui-se que a temperatura ambiente afeta o desempenho dos frangos de corte, porém não altera o número, diâmetro e freqüência de fibras musculares no músculo flexor longo do hálux. A restrição alimentar precoce pode ser adotada como prática de manejo, sem que se observem alterações do desempenho na idade de abate e nem na composição das fibras musculares esqueléticas dos frangos de corte.
\end{abstract}

Palavras-chave: fibra, frango, músculo esquelético, restrição alimentar, temperatura

\section{Effect of Environmental Temperature and Feed Restriction on the Performance and Composition of the Skeletal Muscle Fibers in Broilers}

\begin{abstract}
This experiment aimed to evaluate the effects of environmental temperature and feed restriction on performance and characteristics of fiber types in the flexor hallucis longus muscle of male broilers. Three hundred twenty four five-day-old male chicks were allotted to a split plot design, where the main treatments were three feeding programs (ad libitum, early restriction - 8-14 days; later restriction - 29-35 days) and three environmental temperatures of growth (heat, termoneutral and cold) in a completely randomized factorial $3 \times 3$ schedule. The secundary treatments were the ages of the birds (7, 14,21, 28, 35 and 42 days). Statistical analyses confirmed no interactions among feeding program $\mathrm{x}$ temperature and feeding program $\mathrm{x}$ temperature $\mathrm{x}$ age on the broiler performance. Independently of the feeding program, the temperature of raising affected final weight, weight gain, feed intake and feed:gain ratio. Feeding program affected broiler final weight and weigth gain. Feeding program $\mathrm{x}$ age and temperature $\mathrm{x}$ age interactions affected the weights of the body at slaughter and the right leg muscle. These interactions were not significant for left leg muscle weight, cross-sectional right leg muscle area and right leg muscle weight:body weigth ratio. In conclusion, the environmental temperature affected the performance of broiler, but not the number, frequency and size of the myofibers in the flexor hallucis muscle. Early feed restriction can be utilized as management tool without changing the performance at slaughter age and the fiber composition of flexor hallucis longus muscle of male broilers.
\end{abstract}

Key Words: broiler, feed restriction, fiber, skeletal muscle, temperature

\section{Introdução}

Nas condições comerciais de criação de frangos de corte no Brasil, onde não se trabalha com galpões com ambiente controlado, a maioria das granjas é antiga e inadequada em termos de ambiência e con- forto térmico para as aves, as quais são submetidas ao estresse de temperatura, frio no inverno e calor no verão. Os prejuízos no desempenho zootécnico são imensos e podem ser ainda maiores, se forem consideradas as alterações que podem ocorrer no rendimento e qualidade da carne.

\footnotetext{
${ }_{1}$ Parte da Tese de Doutorado apresentada à FCAV, UNESP, Jaboticabal, SP, pelo primeiro autor. Apoio: FAPESP

2 Prof. Assistente Dr., Depto. Melhoramento e Nutrição, FMVZ, UNESP, Botucatu, SP. E.mail: jrsartori@fca.unesp.br

3 Prof. Dr. Voluntário, Depto. Clínica Veterinária, FMVZ, UNESP, Botucatu, SP. E.mail: elisa.gonzales@uol.com.br

4 Prof. Titular do Depto. Morfologia e Embriologia, IB, UNESP, Botucatu, SP. E.mail: btdalpai@zaz.com.br

5 Prof. Dr. do Depto. Melhoramento e Nutrição, FMVZ, UNESP, Botucatu, SP. E.mail: hnunes@fca.unesp.br

${ }^{6}$ Prof. Titular do Depto. Morfologia e Fisiologia, FCAV, UNESP, Jaboticabal, SP. E.mail: macari@fcav.unesp.br
} 
Assim como em outras espécies domésticas, a musculatura esquelética do frango de corte apresenta três tipos de fibras: brancas, intermediárias e vermelhas. As fibras brancas, de metabolismo anaeróbico, também podem ser denominadas de FG (Fast Glycolytic) e apresentam maior área e contração rápida. As fibras intermediárias, de coloração vermelha, são chamadas de FOG (Fast Oxidative Glycolytic), com área intermediária, metabolismo aeróbico e glicolítico e contração rápida. As fibras vermelhas têm metabolismo aeróbico e são chamadas de SO (Slow Oxidative), tendo pequena área e contração lenta (PETER et al., 1972; BANKS, 1992.).

A presença de um ou mais tipos de fibras, sua distribuição e freqüência dos subtipos, é que determinam as características metabólicas e contráteis do músculo esquelético, revelando suas propriedades bioquímicas e fisiológicas (DALL PAI e CURI, 1992). O fenótipo definitivo de fibras musculares esqueléticas adultas é resultado de eventos que começam no embrião e são modulados no decorrer da vida do organismo. Dessa forma, a composição de fibras musculares em aves pode variar com o tipo de músculo, inervação, localização dentro do músculo, idade, sexo, genética, ambiente (temperatura e dieta), exercícios e doenças (STOCKDALE, 1992; ONO et al., 1993).

Para manter a homeotermia corporal, os frangos de corte produzem calor, no frio, ou dissipam calor, no verão. Esses processos fisiológicos contam com a participação da musculatura esquelética. No frio, o frango de corte procura manter a homeotermia através de aumento na produção de calor, vinculada aos processos vitais e ao tremor muscular, e à redução na perda de calor. Trabalhos têm evidenciado que é possível as aves aumentarem a produção de calor quando expostas ao frio, através do aumento da atividade da enzima Citocromo oxidase, sendo esse efeito associado à musculatura vermelha (MACARI et al., 1994).

DUCHAMP et al. (1992) mostraram que, em paralelo ao desenvolvimento de non shivering thermogenesis em patos aclimatados pelo frio, o músculo esquelético apresentou alterações estruturais e funcionais para aumentar sua capacidade oxidativa, indicado por um aumento na proporção de fibras oxidativas e no suprimento capilar. Outros autores (BALLANTYNE e GEORGE, 1978) observaram que a adaptação ao frio, mas não ao calor, causou diminuição do diâmetro das fibras do músculo peitoral de pombos. Essas observações podem significar que as condições de temperatura do meio ambiente são capazes de alterar a composição das fibras da musculatura esquelética das aves. Há poucas informações com relação à estrutura das fibras musculares de frangos de corte submetidos a temperatura estressante, frio ou calor.

Existem vários estudos com restrição alimentar no período de inverno em frangos de corte em uma idade precoce e por um curto período de tempo, resultando em melhoria na eficiência alimentar, diminuição da gordura na carcaça (PLAVNIK e HURWITZ, 1991) e das perdas causadas por doenças metabólicas (GONZALES, 1992; COELLO et al., 1993; ROBINSON et al., 1989). Outros autores trabalharam com a restrição alimentar em uma fase final de criação dos frangos de corte, com o intuito de diminuir as perdas de desempenho e mortalidade causadas pelo calor nesse período (SARTORI et al., 1997). Em suínos (DAUNCEY e INGRAM, 1988) e em bovinos (ARRIGONI, 1995), a restrição alimentar determinou alterações na composição das fibras musculares esqueléticas, as quais, em bovinos, se mantiveram mesmo depois do período de ganho compensatório. Porém, pouco se conhece sobre os efeitos da restrição alimentar na composição ou freqüência de tipos de fibras musculares esqueléticas em frangos de corte e, conseqüentemente, as alterações que poderão ocorrer na qualidade da carcaça, com ou sem crescimento compensatório.

O objetivo desta pesquisa foi estudar os efeitos da temperatura ambiente (termoneutra, calor e frio) e da restrição alimentar numa fase precoce (8-14 dias) e tardia (29-35 dias) de criação sobre o desempenho e a composição do músculo flexor longo do hálux de frangos de corte.

\section{Material e Métodos}

\section{Criação das aves e delineamento experimental}

O experimento foi realizado no período de $15 \mathrm{de}$ outubro a 26 de novembro de 1997 . Foram utilizados 324 pintos de corte, machos, da linhagem Ross, obtidos de ovos de matrizes de um mesmo lote com 47 semanas de idade. Os ovos foram incubados na mesma incubadora e o nascimento ocorreu em um único nascedouro. Após o nascimento, os pintos foram sexados pela asa, selecionados e vacinados contra Marek, Gumboro e Bouba Aviária. 
As aves foram alojadas com um dia de idade e durante quatro dias permaneceram nas mesmas condições de manejo, alimentação e temperatura ambiente de $35^{\circ} \mathrm{C}$. No quinto dia de idade, as aves foram alojadas em três câmaras climatizadas, iniciando-se o período experimental ( 5 a 42 dias de idade).

O delineamento experimental foi o de parcelas subdivididas, considerando-se os tratamentos principais no esquema fatorial $3 \times 3$ inteiramente casualizado (três programas de alimentação: ad libitum, restrição precoce e restrição tardia e três níveis de temperatura: termoneutra, calor e frio) e os tratamentos secundários, as idades de observação: 7, 14, 21, 28, 35 e 42 dias (MONTGOMERY, 1991).

Foram alojadas 12 aves por boxe em nove boxes por câmara, totalizando 324 aves. Uma câmara foi regulada para manter a termoneutralidade de acordo com a idade da ave; outra câmara foi mantida de 3 a $9^{\circ} \mathrm{C}$ acima da termoneutra e a última câmara foi resfriada a uma temperatura de 7 a $9^{\circ} \mathrm{C}$ abaixo da termoneutra (Tabela 1).

As aves receberam água à vontade durante todo o experimento e, no $11^{\circ}$ dia de idade, foram vacinadas contra a doença de Newcastle (Cepa Lasota), via água de bebida. As rações experimentais (Tabela 2), fornecidas na forma farelada, foram balanceadas para atender as exigências dos frangos de corte em três fases de criação.

A restrição alimentar foi quantitativa, retirando-se $40 \%$ da ração consumida pelas aves alimentadas ad libitum, ajustada diariamente pelo seu consumo diário. A restrição foi aplicada por sete dias na $2^{\mathrm{a}}$ (8-14 dias) e $5^{\mathrm{a}}$ (2935 dias) semanas de idade, denominadas restrição precoce e tardia, respectivamente. Antes e após os períodos de restrição, todas as aves receberam alimentação à vontade.

Tabela 1 - Controle da temperatura ambiente durante o período experimental

Table 1 - Control of environmental temperature during the experimental period

\begin{tabular}{lccc}
\hline Idade (dias) & \multicolumn{3}{c}{ Temperatura ambiente $\left({ }^{\circ} \mathrm{C}\right)$} \\
& \multicolumn{3}{c}{ Environmental temperature $\left({ }^{\circ} \mathrm{C}\right)$} \\
\cline { 2 - 4 } & Termoneutra & Calor & Frio \\
& Termoneutral & Hot & Cold \\
\hline $1-4$ & - & 35 & - \\
$5-7$ & 32 & 35 & 25 \\
$8-14$ & 30 & 34 & 21 \\
$14-16$ & 28 & 34 & 19 \\
$16-21$ & 26 & 33 & 17 \\
$22-42$ & 24 & 33 & 15 \\
\hline
\end{tabular}

Rev. bras. zootec., 30(6):1779-1790, 2001

\section{Avaliações no músculo}

Aos 7, 14, 21, 28, 35 e 42 dias de idade, foram retiradas, ao acaso, três aves de cada tratamento principal, as quais foram sacrificadas por deslocamento cervical. O músculo flexor longo do hálux das pernas direita e esquerda foram dissecados imediatamente após o sacrifício e pesados sem tendões e gordura. Esse músculo foi escolhido, pois tem uma coloração vermelha e apresenta um formato cilíndrico, com distribuição longitudinal das fibras, o que permite medidas morfométricas. Fez-se um corte transversal na região mediana do músculo da perna direita, dividindo-o em duas partes. A parte distal, próxima à articulação tibiotarso-tarsometatarsiana, foi colhida e imediatamente congelada durante dois minutos em $\mathrm{N}-\mathrm{Hexana}$, previamente resfriada a $-70^{\circ} \mathrm{C}$ em $\mathrm{N}_{2}$ líquido (CHAYEN et al., 1969). Os fragmentos foram identificados e acondicionados em botijão de $\mathrm{N}_{2}$ líquido. Em um micrótomo-criostato a $-20^{\circ} \mathrm{C}$ foram obtidas quatro séries de cortes histológicos com $8 \mathrm{~mm}$ de espessura para cada amostra.

Os cortes foram submetidos às seguintes técnicas: Hematoxilina-Eosina (HE); Nicotinamida Adenina Dinucleotídeo Tetrazólio Redutase (NADH-TR), conforme a técnica de Pearse (1972), modificada por DUBOWITZ e BROOKE (1984) e ATPase miofibrilar (m-ATPase), após pré-incubação em meio alcalino $(\mathrm{pH} 10,4)$ e em meio ácido $(\mathrm{pH} \mathrm{4,6)}$ (DUBOWITZ e BROOKE, 1984). Para a nomenclatura dos tipos de fibra foram adotados os critérios de PETER et al. (1972), que as classificam em SO (Slow Oxidative), FOG (Fast Oxidative Glycolytic) e FG (Fast Glycolytic).

O tamanho da fibra foi avaliado pela medida do seu menor diâmetro (DUBOWITZ eBROOKE, 1984). Para medidas de número e tamanho dos tipos de fibras, foram analisados os cortes que reagiram com a m-ATPase, após pré-incubação ácida ( $\mathrm{pH} 4,6)$, que permite a diferenciação dos três tipos de fibras. Dos cortes presentes em cada lâmina, foi escolhido o mais íntegro e o que apresentava um melhor padrão de reação. As reações NADH-TR e m-ATPase, após pré-incubação alcalina serviram como controle para a identificação dos tipos de fibras. A coloração com HE foi realizada para verificar se não havia alterações nas fibras musculares.

Para a contagem, estabelecimento das freqüências (\%) e obtenção do diâmetro dos tipos de fibras, foram analisados dez áreas de 66.420, $109.630 \mathrm{e}$ $204.560 \mathrm{~mm}^{2} /$ ave, para 7,14 e 21 ou mais dias de 
idade, respectivamente, utilizando-se um microscópio óptico comum acoplado a um analisador de imagens (Videoplan - OPTIMAS - USA) e a um computador. Foram contadas e medidas as fibras que se encontravam dentro das áreas.

Para obtenção da área do corte transversal do músculo, foi utilizada uma lupa acoplada ao analisador de imagens (Videoplan - OPTIMAS - USA) e a um computador. O número de fibras no músculo foi obtido multiplicando-se o número médio dos tipos de fibras por área analisada pela área da secção transversal do músculo e dividindo-se pela área analisada (ABERLE e STEWART, 1983).

A análise estatística dos dados de peso médio, ganho de peso médio, consumo médio de ração, conversão alimentar, peso vivo ao abate, peso e área do músculo, relação peso do músculo/peso vivo e da morfometria dos tipos de fibras musculares (número, freqüência e tamanho) foi feita pelo método da análise de variância com o procedimento GLM do pro-

Tabela 2 - Composição das rações segundo a fase de criação

Table 2 - Composition of the experimental diets according to the rearing phase

\begin{tabular}{|c|c|c|c|}
\hline $\begin{array}{l}\text { Composição } \\
\text { Composition }\end{array}$ & $\begin{array}{c}\text { Inicial } \\
(0 \text { a } 21 \text { dias }) \\
\text { Initial } \\
(0-21 \text { days }) \\
\end{array}$ & $\begin{array}{c}\text { Crescimento } \\
\text { (22 a } 35 \text { dias) } \\
\text { Growing } \\
(22-35 \text { days })\end{array}$ & $\begin{array}{c}\text { Final } \\
\text { (36 a } 42 \text { dias) } \\
\text { Finishing } \\
\text { (36- } 42 \text { days) } \\
\end{array}$ \\
\hline $\begin{array}{l}\text { Milho moído } \\
\text { Corn }\end{array}$ & 55,50 & 59,74 & 61,89 \\
\hline $\begin{array}{l}\text { Farelo de soja } \\
\text { Soybean meal }\end{array}$ & 34,92 & 29,77 & 27,18 \\
\hline $\begin{array}{l}\text { Óleo de soja } \\
\text { Soybean oil }\end{array}$ & 4,58 & 5,49 & 5,93 \\
\hline $\begin{array}{l}\text { Núcleo } \\
\text { premix }^{1}\end{array}$ & $5,00^{2}$ & $5,00^{3}$ & $5,00^{4}$ \\
\hline $\begin{array}{l}\text { Total } \\
\text { Valores calculados }\end{array}$ & 100,00 & 100,00 & 100,00 \\
\hline $\begin{array}{l}\text { Calculated values } \\
\text { Energia metabolizável aparente, } \mathrm{kcal} / \mathrm{kg} \\
\text { Apparent metabolizable energy }\end{array}$ & 3050 & 3150 & 3200 \\
\hline $\begin{array}{l}\text { Proteína bruta, } \% \\
\text { Crude protein }\end{array}$ & 21,000 & 19,000 & 18,000 \\
\hline $\begin{array}{l}\text { Gordura, } \% \\
\text { Fat }\end{array}$ & 6,678 & 7,682 & 8,173 \\
\hline $\begin{array}{l}\text { Fibra bruta, } \% \\
\text { Crude fiber }\end{array}$ & 3,244 & 2,987 & 2,858 \\
\hline $\begin{array}{l}\text { Cálcio, \% } \\
\text { Calcium }\end{array}$ & 1,013 & 0,995 & 0,916 \\
\hline $\begin{array}{l}\text { Fósforo disponível, \% } \\
\text { Available phosphorus }\end{array}$ & 0,437 & 0,430 & 0,375 \\
\hline $\begin{array}{l}\text { Metionina }+ \text { cistina disponíveis, } \% \\
\text { Available methionine }+ \text { cystine }\end{array}$ & 0,817 & 0,705 & 0,659 \\
\hline $\begin{array}{l}\text { Metionina disponível, \% } \\
\text { Available methionine }\end{array}$ & 0,471 & 0,386 & 0,354 \\
\hline $\begin{array}{l}\text { Lisina disponível, } \% \\
\text { Available lysine }\end{array}$ & 1,146 & 1,010 & 0,941 \\
\hline
\end{tabular}

${ }_{1}^{1}$ Agromix AC50 - composição básica (quantidade por kg de produto): vitamina (vit.) A 176000 UI, vit. D3 40000 UI, vit. E 600 mg, vit. K3 100 mg, vit. B1 $36 \mathrm{mg}$, vit. B2 $200 \mathrm{mg}$, vit. B6 $50 \mathrm{mg}$, vit. B12 $560 \mathrm{mcg}$, niacina $700 \mathrm{mg}$, biotina $3 \mathrm{mg}$, ácido pantotênico $500 \mathrm{mg}$, ácido fólico $300 \mathrm{mg}$, colina $20 \mathrm{mg}$, ferro $1100 \mathrm{mg}$, cobre $300 \mathrm{mg}$, manganês $1800 \mathrm{mg}$, zinco $1200 \mathrm{mg}$, iodo $24 \mathrm{mg}$, selênio $3 \mathrm{mg}$, metionina $20 \mathrm{~g}$, cálcio $176 \mathrm{~g}$, fósforo $68 \mathrm{~g}$, sódio $23 \mathrm{~g}$, cloro $36 \mathrm{~g}$, antifúngico $200 \mathrm{mg}$ e BHT $1 \mathrm{~g}$

2 Agromix AC50 inicial: acrescenta-se na composição básica $20 \mathrm{~g}$ de Nicarmix e $2 \mathrm{~g}$ de Lincomix.

3 Agromix AC50 crescimento: acrescenta-se na composição básica $10 \mathrm{~g}$ de Coxistac e $2 \mathrm{~g}$ de Lincomix.

4 Agromix AC50 final: composição básica sem anticoccidiano e promotor de crescimento.

1 Agromix AC50 - basic composition (amount per kg the product): vitamin (vit.) a 17600 UI, vit. D3 40000 UI, vit. E 600 mg, vit. K3 100 mg, vit. B1 36 mg, vit. B2 200 $\mathrm{mg}$, vit. B6 $50 \mathrm{mg}$, vit. B12 $560 \mathrm{mcg}$, niacin $700 \mathrm{mg}$, biotin $3 \mathrm{mg}$, pantothenic acid $500 \mathrm{mg}$, folic acid $300 \mathrm{mg}$, choline $20 \mathrm{mg}$, iron $1100 \mathrm{mg}$, copper $300 \mathrm{mg}$, manganese $1800 \mathrm{mg}$, zinc $1200 \mathrm{mg}$, iodine $24 \mathrm{mg}$, selenium $3 \mathrm{mg}$, methionine $20 \mathrm{~g}$, calcium $176 \mathrm{~g}$, phosphorus $68 \mathrm{~g}$, sodium $23 \mathrm{~g}$, chloride $36 \mathrm{~g}$, antifungi $200 \mathrm{mg}$ and BHT $1 \mathrm{~g}$.

2 Agromix AC50 starter: basic composition plus Nicarmix $20 \mathrm{~g}$ and Lincomix $2 \mathrm{~g}$.

3 Agromix AC50 growing: basic composition plus Coxistac $10 \mathrm{~g}$ and Lincomix $2 \mathrm{~g}$.

4 Agromix AC50 finishing: basic composition without antimicrobial feed additives. 
grama SAS (1996). Quando necessário, as médias de temperatura, programa de alimentação e idade foram comparadas pelo teste de Tukey, segundo SAS (1996).

\section{Resultados e Discussão}

\section{Desempenho}

Aos cinco dias de idade, o peso médio dos pintainhos foi 79,09 $\pm 1,76 \mathrm{~g}$. Os dados acumulados de desempenho para o período experimental(5-42 dias) estão descritos na Tabela 3. Não se observou interação significativa entre temperatura e programa de alimentação e nem entre temperatura, programa de alimentação e idade para nenhuma das características de desempenho estudadas.

Aos 42 dias de idade, o peso e o ganho de peso das aves criadas no ambiente quente foram inferiores $(\mathrm{P}<0,05)$ aos das aves mantidas em ambiente termoneutro e frio. Estes resultados ocorreram, provavelmente, devido ao menor $(\mathrm{P}<0,05)$ consumo de ração das aves na câmara aquecida, quando comparadas com as aves criadas nas câmaras termoneutra e fria. Entre as aves alojadas nas câmaras termoneutra e fria, o consumo de ração foi menor $(\mathrm{P}<0,05)$ para aves alojadas na câmara termoneutra, indicando que a temperatura fria provocou um aumento no consumo voluntário de alimento. Estes dados estão de acordo com os observados na literatura. Vários autores têm relatado queda de desempenho em lotes de frangos de corte criados em períodos quentes, em decorrência de menor consumo de ração, com menores ganhos de peso e peso final (TEETER et al.,

Tabela 3 - Resultados médios de peso inicial (PI), peso final (PF), ganho de peso (GP), consumo de ração (CRM) e conversão alimentar (CA) de frangos de corte machos aos 42 dias de idade, segundo a temperatura ambiente e o programa de alimentação

Table 3 - Means of initial body weight (PMI), final body weight (PMF), body weight gain (GPM), feed intake (CR) and feed:gain ratio (CA) of male broilers at 42 days of age, according to environmental temperature and feeding program

\begin{tabular}{|c|c|c|c|c|c|}
\hline & \multicolumn{5}{|c|}{$\begin{array}{l}\text { Parâmetros } \\
\text { Parameters }\end{array}$} \\
\hline & $\mathrm{PI}(\mathrm{g})$ & $\mathrm{PF}(\mathrm{g})$ & GP (g) & $\mathrm{CR}(\mathrm{g})$ & $\mathrm{CA}$ \\
\hline \multicolumn{6}{|c|}{$\begin{array}{l}\text { Temperatura ambiente }(\mathrm{T}) \\
\text { Environmental temperature }\end{array}$} \\
\hline $\begin{array}{l}\text { Quente } \\
\text { Hot }\end{array}$ & $80^{\mathrm{NS}}$ & $1807^{\mathrm{B}}$ & $1727^{\mathrm{B}}$ & $2800^{\mathrm{C}}$ & $1,79^{\mathrm{B}}$ \\
\hline $\begin{array}{l}\text { Termoneutra } \\
\text { Termoneutral }\end{array}$ & 79 & $2271^{\mathrm{A}}$ & $2192^{\mathrm{A}}$ & $3579^{\mathrm{B}}$ & $1,83^{\mathrm{AB}}$ \\
\hline $\begin{array}{l}\text { Fria } \\
\text { Cold } \\
\text { Programa de alime } \\
\text { Feed program }\end{array}$ & 79 & $2310^{\mathrm{A}}$ & $2231^{\mathrm{A}}$ & $4014^{\mathrm{A}}$ & $1,96^{\mathrm{A}}$ \\
\hline $\begin{array}{l}\text { Ad libitum } \\
\text { Ad libitum }\end{array}$ & $79^{\mathrm{NS}}$ & $2196^{\mathrm{A}}$ & $2117^{\mathrm{A}}$ & $3631^{\mathrm{NS}}$ & $1,89^{\mathrm{NS}}$ \\
\hline $\begin{array}{l}\text { Restrição precoce } \\
\text { Early restriction }\end{array}$ & 80 & $2150^{\mathrm{AB}}$ & $2070^{\mathrm{AB}}$ & 3408 & 1,82 \\
\hline $\begin{array}{l}\text { Restrição tardia } \\
\text { Later restriction } \\
\text { F }\end{array}$ & 78 & $2041^{\mathrm{B}}$ & $1963^{\mathrm{B}}$ & 3354 & 1,86 \\
\hline (T) & 1,35 & 43,91 & 43,36 & 59,67 & 5,66 \\
\hline $\mathrm{P}$ & 0,28 & 0,00 & 0,00 & 0,00 & 0,01 \\
\hline (PA) & 1,66 & 3,54 & 3,43 & 3,40 & 0,85 \\
\hline $\mathrm{P}$ & 0,22 & 0,05 & 0,05 & 0,06 & 0,44 \\
\hline (TxPA) & 0,61 & 0,87 & 0,85 & 1,15 & 0,20 \\
\hline $\mathrm{P}$ & 0,66 & 0,50 & 0,51 & 0,37 & 0,93 \\
\hline Médias & 79 & 2129 & 2050 & 3464 & 1,86 \\
\hline $\begin{array}{l}\text { Means } \\
\text { DP } 1\end{array}$ & 2 & 127 & 128 & 239 & 0.11 \\
\hline CV $(\%)^{1}$ & 2,25 & 5,96 & 6,24 & 6,90 & 5,97 \\
\hline
\end{tabular}

$A, B, C$ Médias na coluna seguidas de letras diferentes diferem $(P<0,05)$ pelo teste de Tukey.

NS Não significativo $(P>0,05)$.

$1 \quad \mathrm{DP}=$ desvio-padrão; $\mathrm{CV}=$ coeficiente de variação.

$A, B, C \quad$ Means in a column followed by different letters differ $(P<.05)$ by Tukey test.

NS Non significant effect $(P>05)$.

$1 D P=$ standard deviation; $C V=$ coefficient of variation. 
1989; MAY e LOTT, 1992). Embora não significativos, os valores menores para peso e ganho de peso das aves submetidas à termoneutralidade, quando comparadas ao ambiente frio, estão em conformidade com o NRC (1981), onde relata-se que há influência da temperatura ambiente no desempenho de frangos de corte, observando-se um ganho de peso e consumo de ração máximos na estação fria e mínimos no período quente.

Houve um efeito significativo $(\mathrm{P}<0,05)$ da temperatura ambiente sobre a conversão alimentar, sendo que as aves criadas em ambiente frio apresentaram um índice de conversão alimentar maior $(\mathrm{P}<0,05)$ que as criadas no quente, não diferindo significativamente das aves criadas na termoneutralidade (Tabela 3). DAGHIR (1995) relata que a eficiência alimentar para frangos de corte é máxima aos $27^{\circ} \mathrm{C}$, sendo sempre reduzida em temperaturas abaixo de $21^{\circ} \mathrm{C}$, conforme observado neste experimento.

Aos 42 dias de idade, não houve diferença $(\mathrm{P}<0,05)$ para peso, ganho de peso, consumo de ração e conversão alimentar entre as aves alimentadas ad libitum e as aves submetidas a restrição precoce (814 dias), indicando que as aves submetidas a restrição precoce apresentaram um ganho compensatório após a restrição, recuperando o peso aos 42 dias de idade. GONZALES (1992), trabalhando com níveis de restrição de até $40 \%$ na segunda semana de idade de frangos de corte, não verificou alterações no peso final e consumo de ração acumulado aos 49 dias de idade, indicando que houve um crescimento compensatório, como observado neste experimento.

As aves submetidas à restrição alimentar tardia (2935 dias) tiveram menores peso e ganho de peso aos 42 dias de idade que as aves alimentadas ad libitum. Esses resultados não estão de acordo com os observados por SARTORI et al. (1997), que não verificaram alterações significativas no peso final, ganho de peso e consumo de ração para frangos de corte submetidos ao jejum de até $8 \mathrm{~h} /$ dia na fase final de criação.

Não houve efeito de programa de alimentação para a conversão alimentar, diferindo dos resultados de PLAVNIK e HURWITZ (1991), que verificaram uma melhora na eficiência alimentar com o uso de restrição precoce. Porém, GONZALES (1992), trabalhando com restrição alimentar quantitativa (10 a $50 \%$ ) na segunda semana de idade, também não observou diferenças na conversão alimentar para frangos alimentados ad libitum, quando comparados aos submetidos a $40 \%$ de restrição de 8 a 14 dias de idade, em lotes criados no inverno e no verão.

\section{Morfometria do músculo}

O peso vivo ao abate teve comportamento semelhante ao peso e ganho de peso médios do lote para temperatura e programa de alimentação dentro de cada idade (Tabela 4). Neste experimento, o peso vivo individual da ave ao abate foi sempre superior ao peso médio do lote, em todos os grupos de tratamento. Esses maiores valores numéricos podem ser explicados pela amostragem, onde, muitas vezes, as aves amostradas para o abate são mais pesadas do que o peso médio do lote. Entretanto, avaliações de qualidade de carcaça com aves de um mesmo ciclo de criação, linhagem e idade não são prejudicadas, desde que a variação do peso vivo antes do abate, em relação ao peso médio do lote, seja inferior a três desvios padrões (GONZALES et al., 1998), como verificado neste experimento (dados não mostrados).

Houve interação significativa $(\mathrm{P}<0,05)$ entre temperatura e idade e entre programa de alimentação e idade para o peso do músculo da perna direita. Não foram observadas interações significativas entre esses fatores para peso do músculo da perna esquerda, área da secção transversal do músculo e relação peso do músculo da perna direita/peso vivo. Também não houve interação significativa entre idade, temperatura e programa de alimentação para as características morfométricas estudadas (Tabela 4). O desdobramento da interação entre temperatura e idade para peso do músculo da perna direita mostrou que, dentro deidade, opeso do músculo foi maior $(\mathrm{P}<0,05)$ no ambiente quente que no frio aos 14 dias de idade e menor $(\mathrm{P}<0,05)$ no ambiente quente que no termoneutro aos 28 dias de idade. Porém, não ocorreram diferenças no peso do músculo em função da temperatura aos 7, 21, 35 e 42 dias de idade.

O desdobramento da interação entre programa de alimentação e idade para peso do músculo da perna direita (Tabela 4) mostrou que, dentro de idade, o peso do músculo foi menor nas aves submetidas a restrição precoce, quando comparadas às alimentadas ad libitum aos 14, 21 e 35 dias de idade. Nas aves submetidas à restrição tardia, o peso do músculo foi menor aos 35 dias de idade, quando comparadas às aves alimentadas ad libitum.

Dentro do fator temperatura e do fator programa de alimentação, o peso do músculo da perna direita aos 7 e 14 dias de idade não diferiram entre si para nenhuma das temperaturas e programas de alimentação utilizados. A partir dos 21 dias de idade, o peso do músculo da perna direita foi significativamente maior 
$(\mathrm{P}<0,05)$ para cada período de idade, dentro de todas as temperaturas e programas de alimentação. Independentemente da temperatura e da idade, houve efeito $(\mathrm{P}<0,05)$ de programa de alimentação para $\mathrm{o}$ peso do músculo da perna esquerda, o qual foi menor nas aves submetidas a restrição precoce que nas alimentadas ad libitum (Tabela 4).

Independentemente do programa de alimentação e da idade, houve efeito da temperatura sobre a relação peso do músculo da perna direita/peso vivo, a qual foi maior $(\mathrm{P}<0,05)$ nas aves no ambiente quente, quando comparadas com as aves nos ambientes termoneutro e frio (Tabela 4).

Independentemente de temperatura e programa de alimentação, houve efeito significativo $(\mathrm{P}<0,05)$ da idade para peso do músculo da perna esquerda, área da
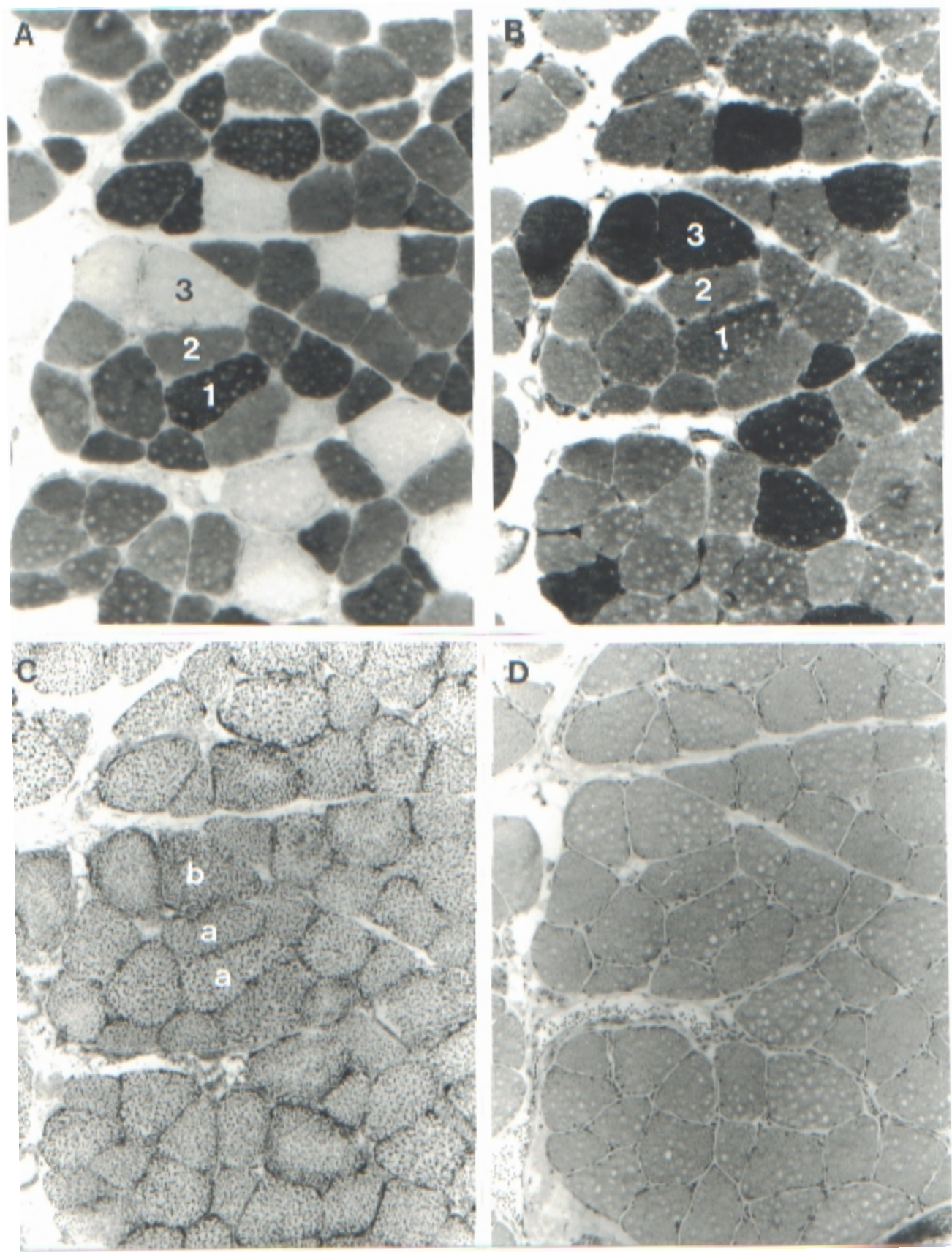

Figura 1 - Cortes transversais do músculo flexor longo do hálux de frangos de corte machos aos 42 dias de idade (A = $m-A T P a s e$, após pré-incubação em pH 4,6; $B=m-A T P a s e$, após pré-incubação em pH 10,4; C = NADH-TR e D = HE). $1=S O$, 2 = FOG e 3 = FG. $a=$ metabolismo oxidativo intenso e $\mathrm{b}=$ metabolismo oxidativo moderado. 400x

Figure 1 - Cross section of flexor hallucis longus of 42-day-old male broilers $(A=m$-ATPase, $p H$ 4,6; $B=m$ - $A T P a s e, p H 10,4 ; C=N A D H$ $T R$ and $D=H E) .1=S O, 2=F O G$ e $3=$ FG. $a=$ intense oxidative metabolism and $b=$ moderate oxidative metabolism. $x 400$. 
Tabela 4 - Resultados médios de morfometria do músculo flexor longo do hálux de frangos de corte machos segundo a temperatura, o programa de alimentação e a idade

Table 4 - Means of morfometric characteristics in the flexor hallucis longus muscle of male broilers according to temperature, feed program, and age

\begin{tabular}{|c|c|c|c|c|c|c|c|}
\hline \multirow{2}{*}{$\begin{array}{l}\text { Idade } \\
\text { (dias) } \\
\text { Age (days) }\end{array}$} & \multicolumn{3}{|c|}{$\begin{array}{l}\text { Temperatura } \\
\text { Temperature }\end{array}$} & \multicolumn{3}{|c|}{$\begin{array}{c}\text { Programa de alimentação } \\
\text { Feed program }\end{array}$} & \multirow[t]{2}{*}{$\begin{array}{l}\text { Médias } \\
\text { Means }\end{array}$} \\
\hline & Q & $\mathrm{TN}$ & $\mathrm{F}$ & $\mathrm{AD}$ & $\mathrm{RP}$ & RT & \\
\hline & \multicolumn{7}{|c|}{$\begin{array}{l}\text { Peso vivo ao abate, } \mathrm{g} \\
\text { Body weigth at slaughter }\end{array}$} \\
\hline 7 & $146^{\mathrm{a}}$ & $144^{\mathrm{a}}$ & $140^{\mathrm{a}}$ & $143^{\mathrm{a}}$ & $144^{\mathrm{a}}$ & $142^{\mathrm{a}}$ & 143 \\
\hline 14 & $311^{\mathrm{a}}$ & $333^{a}$ & $330^{\mathrm{a}}$ & $370^{\mathrm{a}}$ & $254^{\mathrm{b}}$ & $350^{\mathrm{a}}$ & 325 \\
\hline 21 & $639^{b}$ & $668^{a b}$ & $731^{a}$ & $708^{a}$ & $611^{\mathrm{b}}$ & $719^{a}$ & 679 \\
\hline 28 & $982^{\mathrm{b}}$ & $1294^{\mathrm{a}}$ & $1182^{\mathrm{a}}$ & $1222^{\mathrm{a}}$ & $1072^{\mathrm{a}}$ & $1164^{\mathrm{a}}$ & 1153 \\
\hline 35 & $1468^{\mathrm{b}}$ & $1754^{\mathrm{a}}$ & $1732^{\mathrm{ab}}$ & $1838^{a}$ & $1629^{a b}$ & $1488^{b}$ & 1651 \\
\hline 42 & $1889^{\mathrm{b}}$ & $2363^{\mathrm{a}}$ & $2468^{\mathrm{a}}$ & $2331^{\mathrm{a}}$ & $2244^{\mathrm{a}}$ & $2144^{\mathrm{a}}$ & 2240 \\
\hline Médias & 906 & 1093 & 1097 & 1102 & 993 & 1001 & \\
\hline
\end{tabular}

Means

Peso do músculo da perna direita, g Right leg muscle weigth

$\begin{array}{llllllll}7 & 0,08^{\mathrm{a}} & 0,08^{\mathrm{a}} & 0,07^{\mathrm{a}} & 0,08^{\mathrm{a}} & 0,08^{\mathrm{a}} & 0,07^{\mathrm{a}} & 0,08 \\ 14 & 0,22^{\mathrm{a}} & 0,18^{\mathrm{ab}} & 0,17^{\mathrm{b}} & 0,22^{\mathrm{a}} & 0,16^{\mathrm{b}} & 0,20^{\mathrm{a}} & 0,19 \\ 21 & 0,44^{\mathrm{a}} & 0,39^{\mathrm{a}} & 0,39^{\mathrm{a}} & 0,43^{\mathrm{ab}} & 0,36^{\mathrm{b}} & 0,43^{\mathrm{a}} & 0,41 \\ 28 & 0,69^{\mathrm{b}} & 0,83^{\mathrm{a}} & 0,71^{\mathrm{ab}} & 0,76^{\mathrm{a}} & 0,69^{\mathrm{a}} & 0,78^{\mathrm{a}} & 0,74 \\ 35 & 1,12^{\mathrm{a}} & 1,13^{\mathrm{a}} & 1,14^{\mathrm{a}} & 1,29^{\mathrm{a}} & 1,02^{\mathrm{b}} & 1,08^{\mathrm{b}} & 1,13 \\ 42 & 1,46^{\mathrm{a}} & 1,49^{\mathrm{a}} & 1,62^{\mathrm{a}} & 1,58^{\mathrm{a}} & 1,44^{\mathrm{a}} & 1,54^{\mathrm{a}} & 1,52 \\ \text { Médias } & 0,67 & 0,68 & 0,68 & 0,73 & 0,62 & 0,68\end{array}$

Means

Peso do músculo da perna esquerda, $\mathrm{g}$

\begin{tabular}{llllllrr} 
& \multicolumn{5}{c}{ Left leg muscle weigth } & 0,07 & $0,07^{\mathrm{F}}$ \\
7 & 0,08 & 0,07 & 0,07 & 0,07 & 0,08 & $0,19^{\mathrm{E}}$ \\
21 & 0,21 & 0,18 & 0,17 & 0,22 & 0,15 & 0,19 & 0,42 \\
28 & 0,42 & 0,38 & 0,39 & 0,42 & 0,36 & $0,70^{\mathrm{D}}$ \\
35 & 0,68 & 0,83 & 0,73 & 0,76 & 0,70 & $1,74^{\mathrm{C}}$ \\
42 & 1,12 & 1,14 & 1,11 & 1,24 & 1,04 & 1,08 \\
Médias & 1,47 & 1,49 & 1,63 & 1,59 & 1,47 & 1,53 \\
$M$ & $0,66^{\mathrm{ns}}$ & 0,68 & 0,68 & $0,72^{\mathrm{a}}$ & $0,63^{\mathrm{b}}$ & $0,68^{\mathrm{ab}}$
\end{tabular}

Means

\begin{tabular}{|c|c|c|c|c|c|c|c|}
\hline 7 & 9,10 & 9,27 & 9,25 & 8,78 & 10,20 & 8,64 & $9,20^{\mathrm{E}}$ \\
\hline 14 & 16,84 & 16,16 & 16,14 & 18,41 & 13,52 & 17,37 & $16,36^{\mathrm{D}}$ \\
\hline 21 & 30,40 & 22,52 & 22,88 & 26,91 & 23,29 & 25,60 & $25,27^{\mathrm{C}}$ \\
\hline 28 & 35,38 & 36,02 & 35,11 & 32,02 & 38,18 & 36,30 & $35,50^{\mathrm{B}}$ \\
\hline 35 & 38,03 & 39,90 & 44,24 & 40,62 & 39,17 & 42,38 & $40,72^{\mathrm{B}}$ \\
\hline 42 & 44,16 & 50,54 & 45,46 & 48,78 & 45,18 & 46,20 & $46,72^{\mathrm{A}}$ \\
\hline Médias & $29,21^{\mathrm{ns}}$ & 29,07 & 28,85 & $29,46^{\mathrm{ns}}$ & 28,26 & 29,42 & \\
\hline \multicolumn{8}{|l|}{ Means } \\
\hline \multicolumn{8}{|c|}{$\begin{array}{l}\text { Peso do músculo da perna direita/peso vivo } \\
\text { Right leg muscle weigth:body weigth ratio }\end{array}$} \\
\hline 7 & 0,056 & 0,053 & 0,052 & 0,057 & 0,053 & 0,052 & $0,054^{\mathrm{C}}$ \\
\hline 14 & 0,071 & 0,052 & 0,055 & 0,057 & 0,063 & 0,057 & $0,059^{\mathrm{BC}}$ \\
\hline 21 & 0,068 & 0,059 & 0,054 & 0,061 & 0,059 & 0,060 & $0,060^{\mathrm{BC}}$ \\
\hline 28 & 0,071 & 0,064 & 0,060 & 0,063 & 0,066 & 0,065 & $0,065^{\mathrm{AB}}$ \\
\hline 35 & 0,076 & 0,064 & 0,065 & 0,070 & 0,069 & 0,067 & $0,069^{\mathrm{A}}$ \\
\hline 42 & 0,078 & 0,063 & 0,066 & 0,070 & 0,068 & 0,068 & $0,069^{\mathrm{A}}$ \\
\hline Médias & $0,070^{\mathrm{a}}$ & $0,059^{\mathrm{b}}$ & $0,059^{b}$ & $0,063^{\mathrm{ns}}$ & 0,063 & 0,062 & \\
\hline
\end{tabular}

Means

\footnotetext{
1 Temperatura ambiente: quente $(\mathrm{Q})$, termoneutra $(\mathrm{TN})$ e fria $(\mathrm{F}) .{ }^{2}$ Programa de alimentação: ad libitum (AD), restrição precoce (RP) e restrição tardia (RT). ns Não significativo $(P>0,05)$.

$a, b, c \mathrm{Na}$ linha, compara o efeito de temperatura ou programa de alimentação dentro de cada idade através do teste de Tukey (P<0,05).

A,B,C,D,E,F Na coluna, compara o efeito da idade pelo teste de Tukey $(P<0,05)$.

${ }^{1}$ Environmental temperature: hot $(Q)$, termoneutral $(T N)$ and cold $(F){ }^{2}$ Feeding programs: ad libitum $(A D)$, early restriction $(R P)$ and later restriction $(R T)$. $N S$ Not significant effect $(P>.05)$.

$a, b, c$ On the line, compare the effect of temperature or feed program within each age by Tukey test $(P<.05)$.

$A, B, C, D, E, F$ In the column, compare the effect of age by Tukey test $(P<.05)$.
} 
secção transversal do músculo da perna direita e relação peso do músculo da perna direita/peso vivo. $\mathrm{O}$ peso do músculo da perna esquerda foi significativamente maior para cada período de idade. A área da secção transversal do músculo da perna direita aumentou $(\mathrm{P}<0,05)$ significativamente para cada período de idade, exceção feita aos 28 e 35 dias. A relação peso do músculo da perna direita/peso vivo aumentou com a idade, sendo que aos 35 e 42 dias de idade, os valores foram maiores que aos sete dias (Tabela 4).

$\mathrm{Na}$ Tabela 5 observa-se que o peso vivo está positivo e significativamente $(\mathrm{P}<0,05)$ correlacionado com o peso e área do músculo flexor longo do hálux da perna direita, mostrando que esse músculo é um bom indicador para estudos relacionados ao cresci- mento dos frangos de corte.

\section{Morfometria das fibras musculares}

Nos cortes do músculo flexor longo do hálux submetidos a reação com a m-ATPase após pré-

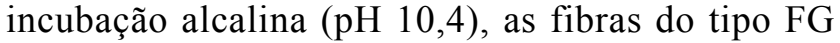
reagiram intensamente e as fibras dos tipos FOG e SO apresentaram uma intensidade de reação de fraca a moderada, de forma que não foi possível diferenciá-las com esta coloração. $\mathrm{Na}$ reação da m-

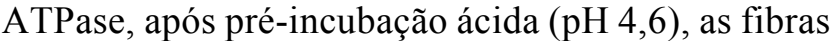
do tipo SO reagiram intensamente, as fibras do tipo FG reagiram fracamente e as fibras do tipo FOG reagiram moderadamente, tendo intensidade de coloração intermediária entre $\mathrm{SO}$ e $\mathrm{FG}$, permitindo a diferenciação dos três tipos de fibras. Na reação com a NADH-TR,

Tabela 5 - Resultados médios de diâmetro e freqüência dos tipos de fibras SO, FOG e FG no músculo flexor longo do hálux de frangos de corte machos segundo a temperatura, o programa de alimentação e a idade

Table 5 - Diameter and frequency means results of SO, FOG and FG fiber types in the flexor hallucis longus muscle of male broilers according to temperature, feeding program and age

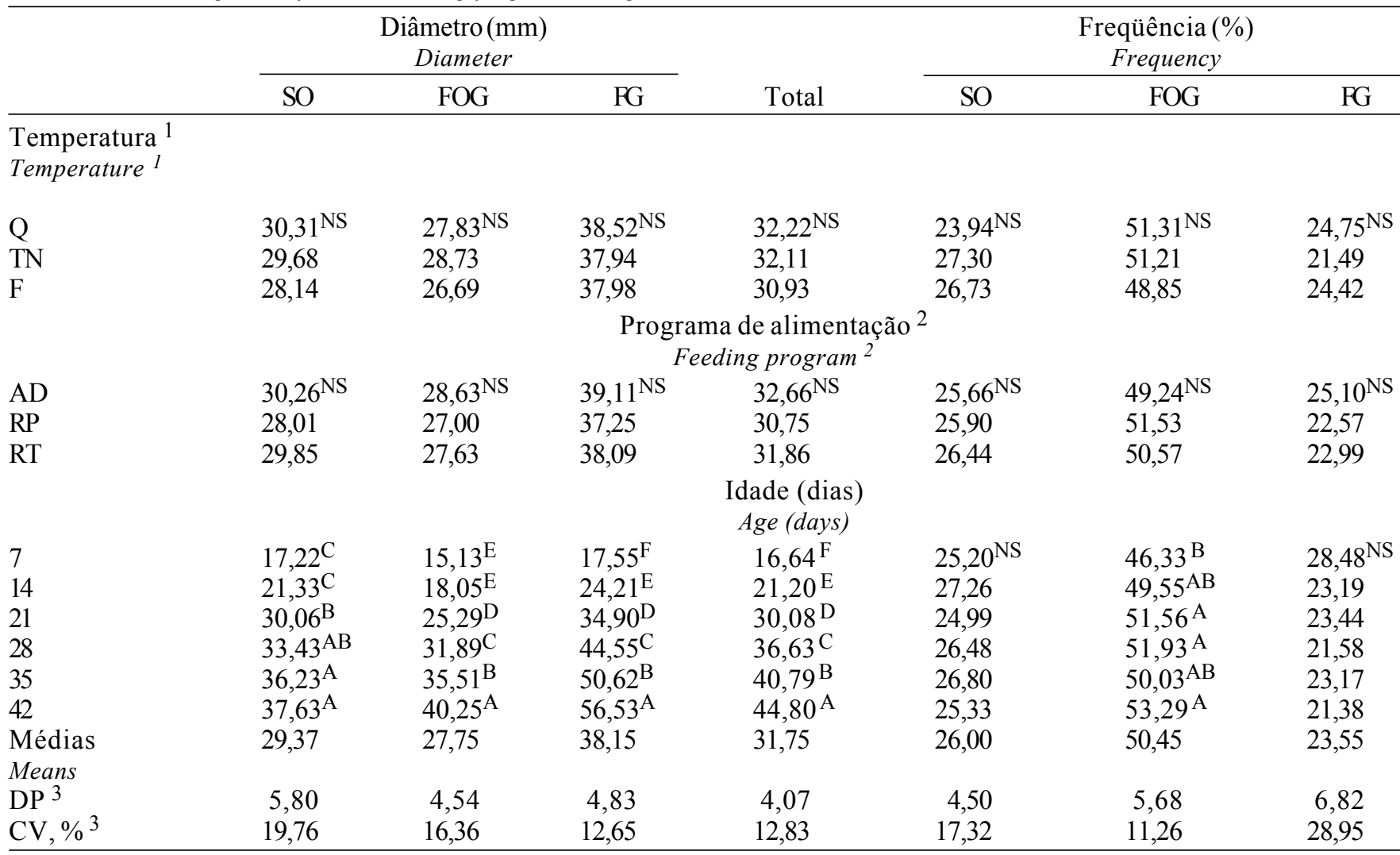

1 Temperatura ambiente: quente $(\mathrm{Q})$, termoneutra $(\mathrm{TN})$ e fria $(\mathrm{F})$.

2 Programa de alimentação: ad libitum (AD), restrição precoce (RP) e restrição tardia (RT).

3 DP = desvio-padrão; $C V$ = coeficiente de variação.

$A, B, C, D, E, F$ Letras diferentes na coluna, dentro de temperatura, programa de alimentação ou idade, indicam diferenças significativas pelo teste Tukey $(P<0,05)$.

NS Não-significativo $(P>0,05)$.

1 Environmental temperature: hot (Q), termoneutral (TN) and cold (F).

2 Feeding programs: ad libitum $(A D)$, early restriction $(R P)$ and later restriction $(R T)$.

3 D.P. = standard deviation; C.V. = Coefficient of variation.

$A, B, C, D, E, F$ Different letters in the column, within temperature, feeding program or age, differ $(P<.05)$ by Tukey test.

NS Non significant effect $(P>$.05). 
Tabela 6 - Coeficientes de correlação entre peso vivo e dados morfométricos do músculo flexor longo do hálux e dos seus tipos de fibras em frangos de corte

Table 6 - Coefficients of correlations between body weight and morfometric values in the flexor hallucis longus muscle and their fiber types of broilers

\begin{tabular}{|c|c|c|c|c|c|c|c|c|c|c|c|c|c|}
\hline & $\mathrm{PV}^{1}$ & PM & AM & DFG & DFO & DSO & DTO & FFG & FFO & FSO & NFG & NFO & NSO \\
\hline$\overline{P M}$ & $0,97 *$ & & & & & & & & & & & & \\
\hline $\mathrm{AM}$ & $0,86^{*}$ & $0,86^{*}$ & & & & & & & & & & & \\
\hline DFG & $0,89 *$ & $0,89 *$ & $0,86^{*}$ & & & & & & & & & & \\
\hline DFO & $0,85^{*}$ & $0,84 *$ & $0,79 *$ & $0,90^{*}$ & & & & & & & & & \\
\hline DSO & $0,69 *$ & $0,70 *$ & $0,73^{*}$ & $0,83^{*}$ & $0,87^{*}$ & & & & & & & & \\
\hline DTO & $0,86^{*}$ & $0,86^{*}$ & $0,84^{*}$ & $0,97 *$ & $0,96^{*}$ & $0,93 *$ & & & & & & & \\
\hline FFG & $-0,22 *$ & $-0,24 *$ & $-0,14$ & $-0,32 *$ & $-0,33^{*}$ & $-0,33^{*}$ & $-0,34 *$ & & & & & & \\
\hline FFO & $0,21 *$ & $0,24 *$ & $0,17^{*}$ & $0,34 *$ & $0,30^{*}$ & $0,41 *$ & $0,36^{*}$ & $-0,77 *$ & & & & & \\
\hline FSO & 0,05 & 0,04 & $-0,02$ & 0,03 & 0,09 & $-0,06$ & 0,02 & $-0,46^{*}$ & $-0,20 *$ & & & & \\
\hline NFG & $0,61 *$ & $-0,22 *$ & 0,02 & $-0,33^{*}$ & $-0,41^{*}$ & $-0,39^{*}$ & $-0,39 *$ & $0,79 *$ & $-0,60 *$ & $-0,38^{*}$ & & & \\
\hline NFO & $-0,18$ & $-0,04$ & $0,23^{*}$ & $-0,09$ & $-0,26^{*}$ & $-0,17^{*}$ & $-0,17 *$ & 0,01 & $0,19 *$ & $-0,28^{*}$ & $0,54^{*}$ & & \\
\hline NSO & 0,32 & $-0,06$ & $0,16^{*}$ & $-0,17 *$ & $-0,29 *$ & $-0,33 *$ & $-0,26^{*}$ & 0,06 & $-0,33 *$ & $0,37^{*}$ & $0,53 *$ & $0,68 *$ & \\
\hline NTO & 0,24 & $-0,12$ & $0,17^{*}$ & $-0,22 *$ & $-0,37 *$ & $-0,33 *$ & $-0,31 *$ & $0,33 *$ & $-0,24 *$ & $-0,19^{*}$ & $0,81 *$ & $0,90 *$ & $0,82 *$ \\
\hline
\end{tabular}

1 Peso vivo (PV), peso do músculo (PM), área do músculo (AM), diâmetro das fibras do tipo FG (DFG), FOG (DFO) e SO (DSO), diâmetro médio dos três tipos de fibras (DTO), freqüência das fibras do tipo FG (FFG), FOG (FFO) e SO (FSO) número das fibras do tipo FG (NFG), FOG (NFO) e SO (NSO) e número total de fibras (NTO) no músculo.

* Significativo $(P<0,05)$.

${ }^{1}$ Body weight (PV), muscle weight (PM), muscle area (AM), diameters of FG (DFG), FOG (DFO) and SO (DSO) fibertypes, mean diameter of fibertypes (DTO), frequency of FG (FFG), FOG (FFO) and FG (FFG) fiber types, number of FG (NFG), FOG (NFO) and SO (NSO) fiber types and total number (NTO) of fibres in the muscle.

* Significant $(P<.05)$.

Tabela 7 - Resultados médios de número de total de fibras (TO) e de cada tipos de fibras (SO, FOG e FG) no músculo flexor longo do hálux de frangos de corte machos, segundo a temperatura, o programa de alimentação e a idade

Table 7 - Means of total fiber number (TO) and number of SO, FOG and FG fiber types in the flexor hallucis longus muscle in the male broilers according to temperature, feed program, and age

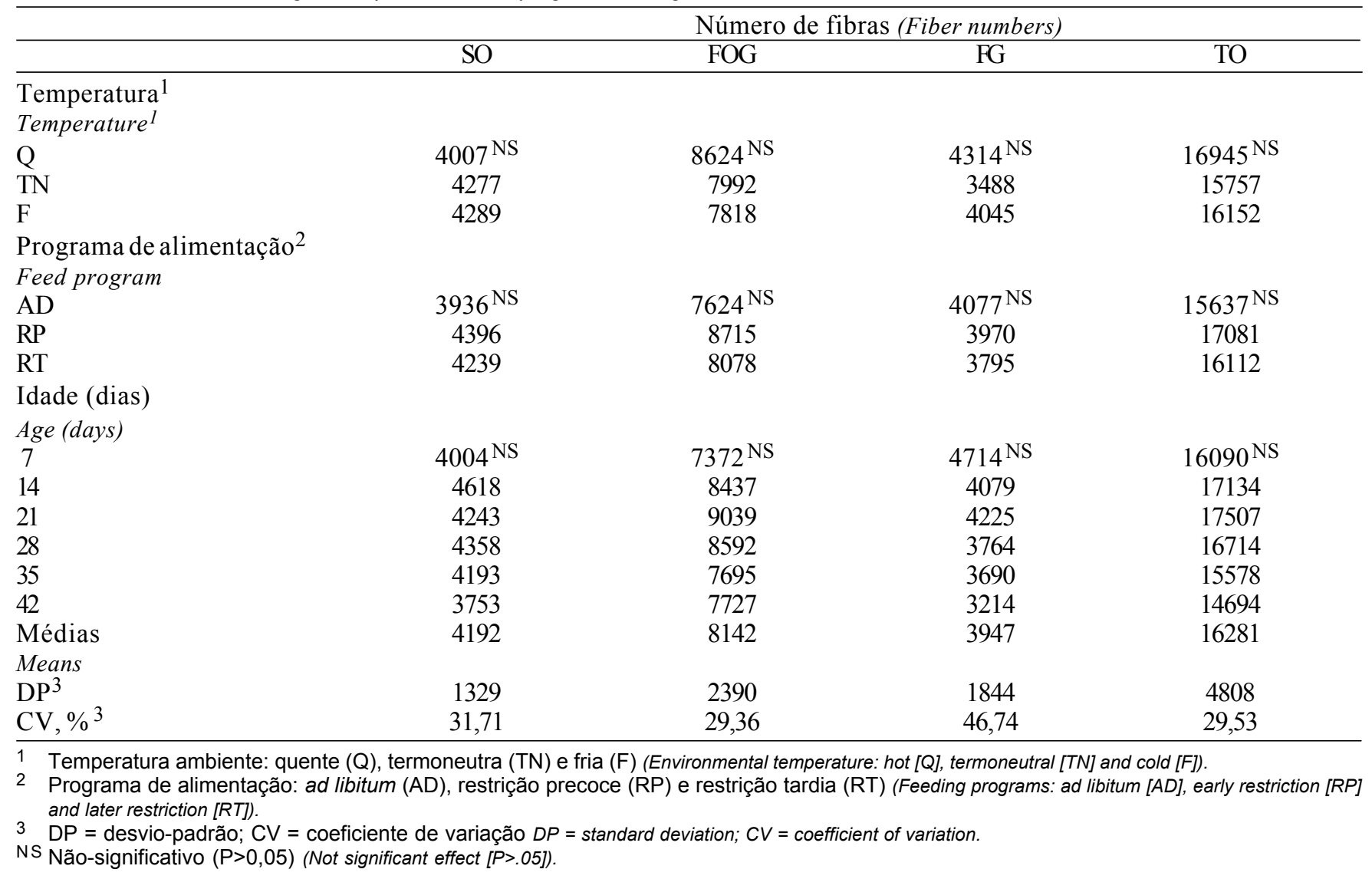


observa-se que as fibras de metabolismo oxidativo (SO e FOG) reagiram intensamente e fibras de metabolismoglicolítico (FG) reagiram moderadamente, indicando que todas as fibras deste músculo apresentavam predomínio de caráter oxidativo (Figura 1).

Não houve interação ou efeito isolado de temperatura e programa de alimentação para os dados de diâmetro e freqüência das fibras musculares do tipo SO, FOG e FG. Também não foram observadas interações significativas entre a idade e a temperatura, entre idade e programa de alimentação e entre idade, temperatura e programa de alimentação para o diâmetro e freqüência para os tipos de fibras musculares analisados (Tabela 6). Esses resultados indicam que a temperatura ambiente fria não influenciou a atividade oxidativa da musculatura, como relatado por BALLANTYNE e GEORGE (1978), em pombos, DUCHAMP et al. (1992), em patos, e MACARI et al. (1994), em aves. A restrição alimentar também não alterou a composição das fibras musculares, diferente do observado por ARRIGONI (1995), em bovinos, e DAUNCEY e INGRAM (1988), em suínos.

Independentemente do programa de alimentação ou da temperatura ambiente, houve efeito significativo $(\mathrm{P}<0,05)$ da idade sobre o diâmetro e a freqüência dos tipos de fibras musculares. O diâmetro das fibras do tipo FG e o diâmetro total das fibras aumentou significativamente $(\mathrm{P}<0,05)$ para cada período de idade. Para as fibras do tipo FOG, não houve diferença entre o diâmetro aos 7 e 14 dias de idade, porém, a partir de 21 dias de idade, o diâmetro aumentou significativamente para cada idade. Para as fibras do tipo SO, não se observou diferença no diâmetro aos 7 e 14 dias de idade, sendo menores que o das fibras aos 21 e 28 dias, que não diferiram entre si. Aos 28, 35 e 42 dias de idade, foram observados os maiores diâmetros das fibras do tipo SO, não diferindo significativamente entre si (Tabela 5).

Os diâmetros das fibras dos tipos SO, FOG e FG foram positivamente $(\mathrm{P}<0,05)$ correlacionados com o peso vivo ao abate e com o peso e a área da secção transversal do músculo flexor longo do hálux da perna direita. Os valores das correlações bastante elevados indicam uma grande contribuição do diâmetro das fibras para o crescimento do animal (Tabela 6).

Aos sete dias de idade, a freqüência de fibras do tipo FOG foi menor que aos 21, 28 e 42 dias, porém não diferiu da freqüência aos 14 e 35 dias. Não houve efeito da idade sobre a freqüência de fibras do tipo SO e FG (Tabela 5).
Não houve efeito de temperatura ambiente, programa de alimentação e idade e nem interações entre estes fatores para o número de fibras do tipo $\mathrm{SO}$, FOG e FG e nem para o número total de fibras no músculo flexor longo do hálux dos frangos de corte (Tabela 7). O número de fibras do tipo SO, FOG e FG e o número total de fibras no músculo flexor longo do hálux foram, em média, 4192, 8142, 3947 e 16281, respectivamente. A temperatura ambiente ou a restrição alimentar não promoveram uma hiperplasia dos tipos de fibras. Isso indica que não há hiperplasia das fibras musculares esqueléticas do músculo flexor longo do hálux durante o período pós-natal em frangos de corte, em conformidade com o observado por ROWE e GOLDSPINK (1969).

\section{Conclusões}

A temperatura ambiente afetou o desempenho dos frangos de corte, porém não alterou o número, diâmetro e freqüência de fibras musculares no músculo flexor longo do hálux. A restrição alimentar precoce pode ser adotada como prática de manejo, sem que se observem alterações do desempenho na idade de abate ou na composição dos tipos de fibras no músculo flexor longo do hálux de frangos de corte.

\section{Referências Bibliográficas}

ABERLE, E.D., STEWART, T.S. 1983. Growth of fiber types and apparent fiber number in skeletal muscle of broiler- and layer- type chickens. Growth, 47:135-144.

ARRIGONI, M.B. Efeito da restrição alimentar sobre o desempenho, área e tipos de fibras musculares em bovinos jovens confinados. Jaboticabal, SP: FCAV, 1995. 73p. Tese (Doutorado em Zootecnia) - Faculdade de Ciências Agrárias e Veterinárias/Universidade Estadual Paulista, 1995.

BALLANTYNE, J.S., GEORGE, J.C. 1978. An ultrastructural and histological analysis of the effects of cold acclimation on vertebrate skeletal muscle. J. Thermal Biol., 3:109-116.

BANKS, W.J. 1992. Histologia veterinária aplicada. 2.ed. São Paulo: Manole Ltda. cap.13. p.215-236.

CHAYEN, J., BITENSKY, L., BUTCHER, R.G. et al. 1969. A guide to pratical histochemstry. London: Oliver \& Bould. 261p.

COEllo, C.L., MENOCAL, J.A., GOnZÁleZ, E.A. Programas de alimentacion utilizados en Mexico para la prevencion y control del sindrome ascitico. In: CONFERÊNCIA APINCO DE CIÊNCIA E TECNOLOGIA AVÍCOLAS, 1993, Santos. Anais... Campinas: FACTA, 1993. p.267-280.

DALL PAI, V., CURI, P.R. 1992. Crescimento pós-natal do coelho Norfolk: correlação entre parâmetros somáticos e área dos tipos de fibras musculares. Pesq. Agropec. Bras., 27:1623-1633. 
DAUNCEY, M.J., INGRAM, D.L. 1988. Influence of environmental temperature and energy intake on skeletal muscle respiratory enzimes and morphology. Eur. J. Appl. Physiol., 58:239-244.

DAGHIR, N.J. 1995. Poultry production in hot climates. Cambridge: University Press. 303p.

DUBOWITZ, V., BROOKE, M. 1984. Muscle biopsy: a modern approach. London: W.B. Saunders Company Ltda. 472p.

DUCHAMP, C., COHEN-ADAD, F., ROUANET, J.L. et al. 1992. Histochemical arguments for muscular non-shivering thermogenesis in muscovy ducklings. J. Physiol., 457:27-45.

GONZALES, E. Estudo da sindrome de morte súbita em frangos de corte. Botucatu, SP: FCAV, 1992. 128p. Tese (Doutorado em Zootecnia) - Faculdade de Ciências Agrárias e Veterinárias/Universidade Estadual Paulista, 1992.

GONZALES, E., GARCIA, E.A., TAKITA, T.S. et al. 1998. Influência do peso vivo de frangos de corte no rendimento de carcaça e partes. Ars Vet., 14:43-49.

MACARI, M., FURLAN, R.L., GONZALES, E. 1994. Fisiologia aviária aplicada a frangos de corte. Jaboticabal: Funep. 296p.

MAY, J.D., LOTT, B.D. 1992. Feed and water consumption patterns of broilers at hight environmental temperatures. Poult. Sci., 71:331-336.

MONTGOMERY, D.C. 1991. Design and analysis of experiments. 3.ed. New York: John Wiley. 649p.

NATIONAL RESEARCH COUNCIL - NRC. 1981. Effect of environment on nutrient requeriments of domestic animals. Washington: National Academic Press. 152p.

ONO, Y., IWAMOTO, H., TAKAHARA, H. 1993. The relationship between muscle growth and the growth of different fiber types in the chicken. Poult. Sci., 72:568-576.

PETER, J.B., BARNARD, R.J., EDGERTON, V.R. et al. 1972. Metabolic profiles of three fiber types of skeletal muscle in Guinea pig and rabbits. Biochemistry, 11:2627-2633.
PLAVNIK, I., HURWITZ, S. 1991. Response of broiler chickens and turkey poults to food restriction of varied severity during early life. Br. Poult. Sci., 32:343-352.

ROBINSON, F.E., HARDIN, R.T., SCOTT, T.A. Short-term feed restriction for broiler and roaster chickens. In: ANNUAL FEEDER'S DAY REPORT AGRICULTURA AND FORESTRY BULLETIN SPECIAL ISSUE, 68, 1989, Alberta. Proceedings... Alberta: University of Alberta, 1989. p.45-46.

ROWE, R.W.D., GOLDSPINK, G. 1969. Muscle fibre growth in five different muscle of both sexes of mice. I. Normal mice. J. Anat., 104:519-530.

SARTORI, J.R., GONZALES, E., SOUZA, E.M. et al. 1997. Efeito do período de jejum na fase final de criação de frangos de corte machos sobre o desempenho e mortalidade. R. Bras. Zootec., 26:1192-1199.

SAS Institute Inc., SAS/STAT. User's guide, version 6.11. 4.ed. Cary: Sas Institute Inc., 1996. 842p.

STOCKDALE, F.E. 1992. Miogenic cell lineages. Dev. Biol., 154:284-298.

TEETER, R.G., WIERNUSZ, C., BERLAY, T. et al. 1989. Broilers exposed to acute heat distress are manageable. Feedstuffs, 61:18-25. 